\title{
Effective Visualization and Navigation in a Multimedia Document Collection Using Ontology
}

\author{
Surjeet Mishra and Hiranmay Ghosh \\ TCS Innovation Labs Delhi, Tata Consultancy Services \\ \{surjeet.mishra, hiranmay.ghosh\}@tcs.com
}

\begin{abstract}
We present a novel user interface for visualizing and navigating in a multimedia document collection. Domain ontology has been used to depict the background knowledge organization and map the multimedia information nodes on that knowledge map, thereby making the implicit knowledge organization in a collection explicit. The ontology is automatically created by analyzing the links in Wikipedia, and is delimited to tightly cover the information nodes in the collection. We present an abstraction of the knowledge map for creating a clear and concise view, which can be progressively 'zoomed in' or 'zoomed out' to navigate the knowledge space. We organize the graph based on mutual similarity scores between the nodes for aiding the cognitive process during navigation.
\end{abstract}

\section{Introduction}

The retrieval tools and classification hierarchy at digital libraries do not provide a collection overview, making exploration difficult for novice or casual users. A user is often lost in the document collection that can be thought as a network of large number of information nodes in different media forms (text, image, video, etc.). The user, interested in information pertaining to a set of related topics, may not have sufficient subject knowledge to locate it on the knowledge map. An efficient visualization tool allows the user to explore the collection by direct interacting with the view. We present a novel user interface that facilitates visualization of and navigation in large multimedia collection.

Earlier work on collection visualization [1,2,3] (re)-organize the document collection to create different views of the collection based on classical distance measures of information retrieval theories. These measures can be computed for text documents alone and cannot be extended predominantly to multimedia collections. Moreover, these visualization schemes cluster document collections based on inter-document distance measures. The semantic relations between the clusters remain unexplored. We consider the underlying knowledge organization structure is important for visualizing a collection. We propose construction of an ontological map of the collection and map the document nodes on it, thereby making the implicit knowledge organization in the collection explicit. The ontology is automatically created by analyzing the links in a public knowledge resource, namely the Wikipedia, and is delimited to tightly cover the information nodes in the collection. The resulting ontology contains several thousand nodes, which cannot be meaningfully visualized together. We have 
developed a new method to present an abstraction of the knowledge map, which can be progressively 'zoomed in' or 'zoomed out' to navigate in the knowledge space. Several approaches to visualize large ontology have been presented in [4] and [5]. Our approach is unique in that it presents abstract views of the knowledge structure at different levels, does not clutter the display with too many nodes and enables flexible zoom and navigation operations. Starting with the overall structure of the collection, a user can deep-dive into some broad areas of interest, progressively refine his information needs and contextually discover the documents in the collection. Our main claims in this paper are (1) automatic organization of the information nodes present in a collection in an ontological structure, and (2) intelligently creating and presenting suitable abstractions of information structure to facilitate the navigation process. A prototype has been developed with a collection of over 650 book and documentaries taken from a multimedia document collection ${ }^{1}$.

The paper is organized as follows. Section 2 describes our work in detail. Section 3 has implementation details and illustrative examples. Section 4 concludes the paper.

\section{Description of the Work}

Our work has two major components, namely (a) creating ontology from Wikipedia, covering all information nodes in a collection, and (b) providing abstract views and navigation facility in the collection with the ontology.

\subsection{Ontology Creation}

For effective visualization and navigation, the documents in the collection need to be interrelated on a knowledge space. Ontology is a formal tool to represent a bounded knowledge domain. Research in informal ontology (or folksonomy) [6] has assumed significance because of the challenges in creating formal ontology for an ill-defined domain like the collection of a bookstore. The categorization structure of an online public encyclopedia, e.g. Wikipedia, represents an informal knowledge organization. Our use of Wikipedia to create an informal ontology is motivated by [7]. We have created a constrained ontology by restricting it to contain the category nodes, sufficient to cover the information nodes in the collection. We treat the category node "Main topic classifications" (MTC), which cover all Wikipedia topics (articles), as the root node of our ontology.

We assume sufficient metadata is associated with each document, to relate the document to one or more Wikipedia topic. Once the topics pertaining to a document have been identified, we create the ontology superstructure for that document in a bottom-up manner starting from a topic and going up the Wikipedia category hierarchy ladder using Depth First Traversal, till we reach the MTC node. This process is repeated for every topic in a document and for every document in the collection, merging the common sub-graph as soon as it is discovered. Thus, a document is associated with one or more leaf category nodes in the ontology (Fig.1). The incremental way of building helps in updating the ontology when new documents are added to a collection.

${ }^{1}$ We thank 'Scholars Without Borders', an online bookstore for scholarly publications, for making its collection available for this research. 


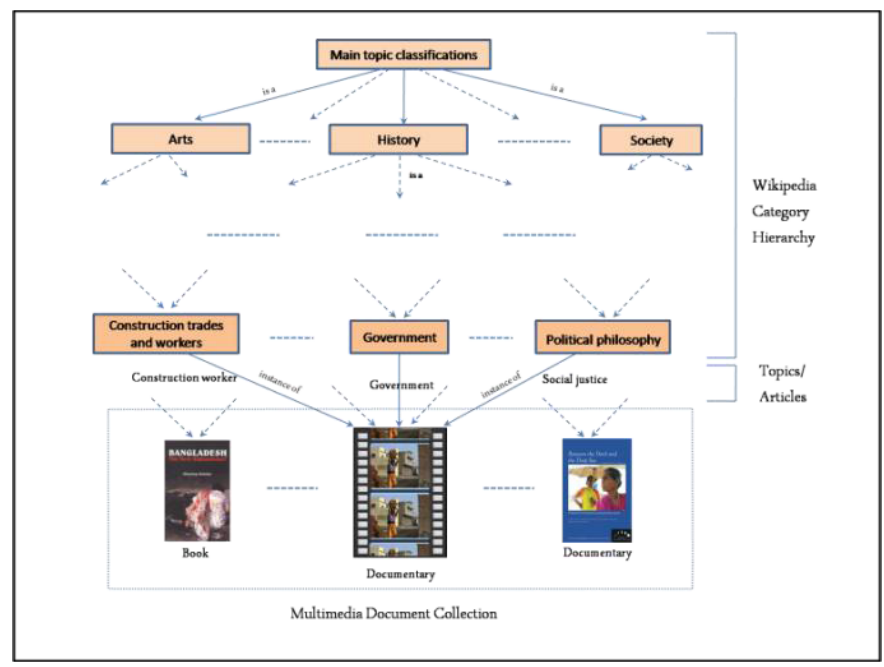

Fig. 1. Knowledge organization in Ontology

We represent the knowledge graph as a singly rooted Directed Acyclic Graph (DAG), with MTC node as the root, to restrict the user from looping in cycles during navigation. We have discovered and removed some cycles in Wikipedia categorization (e.g. Psychology $\rightarrow$ Behavior $\rightarrow$ Human behavior $\rightarrow$ Psychology) by backtracking when an edge completes a loop and by removing the Orphan Nodes ${ }^{2}$.

Thus, the ontology created by us is represented by a singly rooted $\mathrm{DAG}<\mathrm{V}, \mathrm{E}>$, where set of vertices comprise a set of documents $\mathrm{D}$ and set of categories $\mathrm{C}$. The set $\mathrm{E}$ comprises two types of edges: (a) "is-a", that connects a category node to another, and (b) "instance-of", that connects a document node to a category node. We represent these relations by the symbols ' $\subset$ ' and ' $\langle$ ' respectively. Note that the document nodes appear as the leaf nodes. We observe the following transitive properties:

1. Let $\mathrm{c} 1, \mathrm{c} 2, \mathrm{c} 3 \in \mathrm{C}$. If $\mathrm{c} 1 \subset \mathrm{c} 2$ and $\mathrm{c} 2 \subset \mathrm{c} 3$, then $\mathrm{c} 1 \subset \mathrm{c} 3$

2. Let $\mathrm{c} 1, \mathrm{c} 2 \in \mathrm{C}, \mathrm{d} \in \mathrm{D}$. If $\mathrm{d}\langle\mathrm{c} 1$ and $\mathrm{c} 1 \subset \mathrm{c} 2$, then $\mathrm{d}<\mathrm{c} 2$

These properties has been used to abstract the knowledge map at multiple levels and to allow 'zoom-in' and 'zoom-out' operations, that is explained in next sub-section.

\subsection{Navigation Interface}

The knowledge map so created contains a few thousands of category nodes, posing a challenge to visualization, though several large graph visualization techniques have been developed. The folding and unfolding of subject taxonomies [4] provides a shallow overview of the knowledge organization and may pose a significant navigational challenge to a casual user. Hyperbolic distortion $[4,8]$ present nodes of interest at the center of view with magnification, while pushing the other nodes to the periphery which prevents visualizing the overall knowledge structure. Another approach is clustering of nodes $[3,4]$, where semantic labeling of the clustered nodes is a challenge.

\footnotetext{
${ }^{2}$ Category node with no parent category.
} 
We present an abstract view of the ontology with a few selected category nodes and edges that depicts the overall structure of the graph. We compute Strahler Score [9], which is a measure of structural complexity of the sub-graph below the node, for all the category nodes in the graph and select a set of top-ranking nodes. The number of nodes selected depends on the area allocated for visualizing the graph. Let the set of these summary nodes be designated by $V^{\prime}$. We construct the summary graph by drawing summary edges $\mathrm{v}_{1} \rightarrow \mathrm{v}_{2}\left(\mathrm{v}_{1}, \mathrm{v}_{2} \in \mathrm{V}^{\prime}\right)$ if there is a path from $\mathrm{v}_{1}$ to $\mathrm{v}_{2}$, since both the semantic relationships are transitive.

For a graph $\mathrm{G}=\langle\mathrm{V}, \mathrm{E}\rangle$, where $\mathrm{V}$ and $\mathrm{E}$ are set of vertices and edges respectively, and a given set of vertices $V^{\prime}$, the Summary Graph will be $G^{\prime}=\left\langle V^{\prime}, E^{\prime}\right\rangle$ where,

$$
\begin{aligned}
& \mathrm{V}^{\prime} \subseteq \mathrm{V} \text {, and } \\
& \mathrm{E}^{\prime}=\left\{\left(\mathrm{v}_{1} \rightarrow \mathrm{v}_{2}\right) \mid\left(\mathrm{v}_{1}, \mathrm{v}_{2} \in \mathrm{V}^{\prime}\right)\right. \text { AND } \\
& \left.\left.\quad\left(\exists \text { path } \mathrm{p} \text { from } \mathrm{v}_{1} \text { to } \mathrm{v}_{2} \text { in } \mathrm{G} \mid ! \exists \mathrm{v}_{3} \in \mathrm{p}, \mathrm{v}_{3} \in \mathrm{V}^{\prime}\right)\right)\right\}
\end{aligned}
$$

Wikipedia category nodes show high degree of interconnectivity. The large amount of edges present in the summary graph clutters the view. So, we create a minimum weighted DAG around the selected nodes by using Chu-Liu/ Edmond's algorithm $[10,11]$ we get the abstract view graph $\mathrm{G}^{\prime \prime}=\left\langle\mathrm{V}^{\prime}, \mathrm{E}^{\prime \prime}\right\rangle$ where $\mathrm{E}^{\prime \prime} \subseteq \mathrm{E}^{\prime}$. Visualization is further aided by placing the category nodes with higher semantic similarities closer to each other. The similarity of the nodes are measured with Jaccard's coefficient [12]

$$
\operatorname{Sim}\left(\mathrm{v}_{\mathrm{a}}, \mathrm{v}_{\mathrm{b}}\right)=\mathrm{P}\left(\mathrm{S}_{\mathrm{a}} \cap \mathrm{S}_{\mathrm{b}}\right) / \mathrm{P}\left(\mathrm{S}_{\mathrm{a}} \cup \mathrm{S}_{\mathrm{b}}\right)
$$

where, $S_{a}, S_{b}$ are the set of descendent nodes of selected nodes $v_{a}, v_{b}$ respectively and $\mathrm{P}(\mathrm{S})$ is cardinality of set $\mathrm{S}$. The user can click on any of the nodes depicted on the screen to navigate on the ontology graph. As a user zooms in, the topics below the selected node gets magnified, the topics in the other regions shrink and some of the dynamically created document clusters split. Thus, a contextual view of the collection is dynamically created as a user navigates the collection. Completeness of the summary view demands that all the category nodes in the original graph should be navigable. To guarantee completeness, we add the immediate parent and child nodes of the node currently being explored to the dynamic knowledge graph. Once the category graph is organized, the cluster of document nodes is attached to each category node signifying the number of documents related to that category.

\section{Implementation Details and Illustrative Examples}

We have created the knowledge graph for well over 650 documents from a multimedia collection using JWPL ${ }^{3}$. Fig. 2 shows the increase in number of ontology nodes with increase in the number of documents in the collection. The number of ontology nodes tends to saturate at a finite value, after sufficient number of documents are inducted, thereby ascertaining tractability of the problem.

\footnotetext{
${ }^{3}$ Java Wikipedia Library, http://www.ukp.tu-darmstadt.de/software/jwpl/
} 


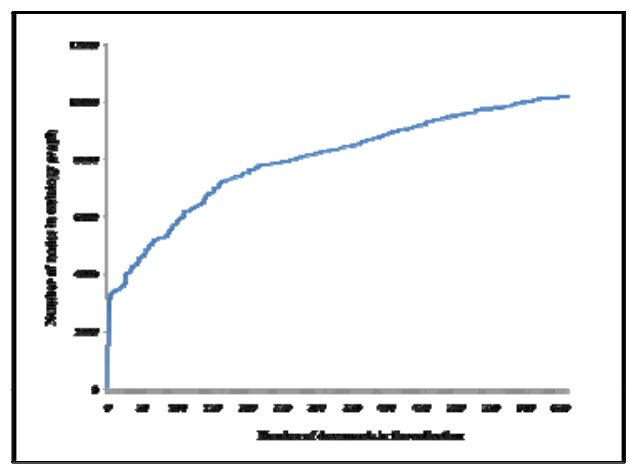

Fig. 2. Increase in number of nodes in ontology with number of documents in the collection

The graph visualization has been implemented with JUNG $2.0^{4}$. We illustrate the navigational steps with a few illustrative examples.

1. To discover the documents ("History and Ideology" and "India") related to tapestry, a user may traverse the path:

MTC $\rightarrow$ Arts $\rightarrow$ Visual arts $\rightarrow$ Textile arts $\rightarrow$ Tapestries

2. To discover documents on Tourism in India, a user traverses the path:

MTC $\rightarrow$ Geography $\rightarrow$ Geography by place $\rightarrow$ Geography by countries $\rightarrow$

Members of the Commonwealth of Nation $\rightarrow$ India $\rightarrow$ Tourism in India

Another interesting aspect of the traversal is user's shift of attention. For example, a user while studying the document "They who walks the mountain" (TWWTM), which speaks about the "life-style of Himalayan people", may be tempted to find more documents on the religious aspect (Buddhism) and end up reaching a document "The Dalai Lama" (TDL). The corresponding navigation path can be:

TWWTM $\rightarrow$ Life in Himalaya $\rightarrow$ Religion $\rightarrow$ Buddhism $\rightarrow$ TDL Some of the navigational steps are shown in Fig. 3.
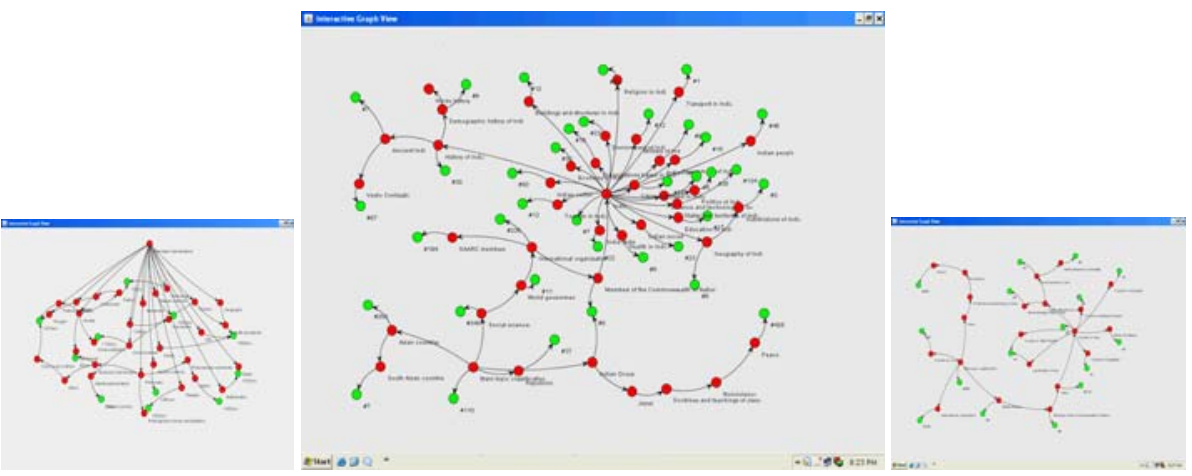

Fig. 3. Abstract views of: (a) overview of ontology (b) intermediate navigation stage (c) final view on "Tourism in India"

\footnotetext{
${ }^{4}$ Java Universal Network/Graph Framework, http://jung.sourceforge.net/index.html
} 
The red bubbles indicate the category nodes and the green bubbles indicate the cluster of associated documents to that category, labeled by the number of documents in the cluster. The cluster expands on clicking and each document can then be accessed.

\section{Conclusion}

The work presented in this paper provides a novel interface for visualization and navigation in a multimedia collection using automatically created ontology. This work can be used to build various applications dealing with multimedia assets in libraries; online book stores, movie stores; video sharing portals; etc. The system can be extended to work with document segments (scenes of video and chapters from literature artifacts) as it can handle any form of information nodes.

\section{References}

1. Grobelnik, M., Mladenic, D.: Efficient visualization of large text corpora. In: Proceedings of the Seventh TELRI seminar, Dubrovnik, Croatia (2002)

2. Paulovich, F.V., Minghim, R.: HiPP: A Novel Hierarchical Point Placement Strategy and its Application to the Exploration of Document Collections. IEEE Transactions on Visualization and Computer Graphics 14(6) (2008)

3. Berendonck, C.V., Jacobs, T.: Bubbleworld- A New Visual Information Retrieval Technique. In: Australasian Symposium on Information Visualisation (2003)

4. Katifori, A., Halatsis, C., Lepouras, G., Vassilakis, C., Giannopoulou, E.: Ontology Visualization Methods-A Survey. ACM Computing Surveys 39(4) (2007)

5. Geroimenko, V., Chen, C. (eds.): Visualizing the Semantic Web-XML-Based Internet and Information Visualization, 2nd edn. Springer, Heidelberg (2006)

6. Hepp, M., Bachlechner, D., Siorpaes, K.: Harvesting Wiki Consensus - Using Wikipedia Entries as Ontology Elements. Web Semantics: Science, Services and Agents on the World Wide Web 6(3), 203-217 (2008)

7. Suchanek, F.M., Kasneci, G., Weikum, G.: Yago: A Large Ontology from Wikipedia and WordNet (2008)

8. Heymann, S., Tham, K., Kilian, A., Wegner, G., Rieger, P., Merkel, D., Freytag, J.C.: Viator - A Tool Family for Graphical Networking and Data View Creation. In: Proc. of the 28th VLDB Conference, Hong Kong, China (2002)

9. Delest, M., Herman, I., Melancon, G.: Tree Visualization and Navigation Clues for Information Visualization. Computer Graphics Forum 17(2) (1998)

10. Chu, Y.J., Liu, T.H.: On the shortest arborescence of a directed graph. Science Sinica 14, 1396-1400 (1965)

11. Edmonds, J.: Optimum branchings. Journal of Research of the National Bureau of Standards 71B, 233-240 (1967)

12. Doan, A., Madhavan, J., Domingos, P., Halevy, A.: Learning to map between ontologies on the semantic web. In: Proc. of the 11th International WWW Conference (2002) 